\title{
Entre Vegas y Valdivia: la fase San Pedro en el suroeste del Ecuador
}

Entre Vegas et Valdivia: la phase San Pedro dans le sud-ouest de l'Équateur Between Vegas and Valdivia: the San Pedro phase in southwestern Ecuador

Henning Bischof y Julio Viteri Gamboa ( $($ )

\section{(2) OpenEdition}

Edición electrónica

URL: http://journals.openedition.org/bifea/3922

DOI: $10.4000 /$ bifea.3922

ISSN: 2076-5827

Editor

Institut Français d'Études Andines

Edición impresa

Fecha de publicación: 1 diciembre 2006

Paginación: 361-376

ISSN: 0303-7495

\section{Referencia electrónica}

Henning Bischof y Julio Viteri Gamboa (†), «Entre Vegas y Valdivia: la fase San Pedro en el suroeste del Ecuador », Bulletin de I'Institut français d'études andines [En línea], 35 (3) | 2006, Publicado el 01 junio 2007, consultado el 01 diciembre 2020. URL : http://journals.openedition.org/bifea/3922 ; DOI : https://doi.org/10.4000/bifea.3922

\section{(c) $(1)$}

Les contenus du Bulletin de l'Institut français d'études andines sont mis à disposition selon les termes de la licence Creative Commons Attribution - Pas d'Utilisation Commerciale - Pas de Modification 4.0 International. 


\title{
Entre Vegas y Valdivia: la fase San Pedro en el suroeste del Ecuador
}

\author{
Henning Bischof* \\ Julio Viteri Gamboa $(t)$
}

\begin{abstract}
Resumen
Un tema que ha quedado en suspenso por algún tiempo, es la introducción de la cerámica en la costa del Ecuador. Con su excavación en Valdivia, los autores trataron de comprobar y ampliar las observaciones previas de Emilio Estrada (Estrada, 1961).

Sobre un estrato precerámico, se descubrió una nueva fase cerámica — San Pedro —, demostrablemente pre-Valdivia ya que los estratos que la arrojan en forma exclusiva, fueron cortados a su vez por un pozo del Valdivia 1. Se discutirán las opiniones emitidas por otros autores sobre estos hallazgos, a la luz de los datos actuales.
\end{abstract}

Palabras clave: arqueología, cerámica formativa, Valdivia, San Pedro, Ecuador

\section{Entre Vegas et Valdivia: la phase San Pedro dans le sud-ouest de l'Équateur}

\section{Résumé}

Un thème peu traité ces dernières années est celui de l'apparition de la céramique sur la côte de l' Équateur. Grâce aux fouilles de Valdivia les auteurs ont tenté de vérifier les observations faites par E. Estrada (Estrada, 1961) et d'en savoir davantage. Au-dessus d'une couche précéramique, une nouvelle phase céramique - San Pedro- a été découverte ; il est possible de démontrer qu'elle est pré-Valdivia puisque les couches qui contiennent la céramique San Pedro sont perforées par une fosse de l'époque Valdivia 1. Dans cet article les opinions d'autres auteurs sont discutées à la lumière des données actuelles.

Mots clés : archéologie, céramique formative, Valdivia, San Pedro, Équateur 


\title{
Between Vegas and Valdivia: the San Pedro phase in southwestern Ecuador
}

\begin{abstract}
The introduction of pottery on the coast of Ecuador has attracted little attention for some time. In order to confirm and expand previous results by Emilio Estrada (Estrada, 1961), the authors extended his original cut «J» at the Valdivia site.

Above basal preceramic layers, a new pottery fase - San Pedro- was discovered. The strata that only yielded this type of pottery are demonstrably pre-Valdivia because they were cut by a pit containing Valdivia 1 pottery (Hill, 1972-1974). These results have been referred to by several authors whose opinions are discussed here on the basis of present data.
\end{abstract}

Key words: archaeology, Formative ceramics, Valdivia, San Pedro, Ecuador

\section{ANTECEDENTES}

Han pasado cincuenta años desde que Emilio Estrada (1956) descubriera la cultura Valdivia, así llamada por lo que entonces fue su mayor yacimiento conocido (fig. 1). Los elementos principales de esta «fase» nueva fueron dados a conocer tres años más tarde (Evans et al., 1959). Quedó por definirse su periodo inicial, tarea que Estrada abordó con la excavación del corte J en la parte más antigua del sitio. El trabajo de campo fue conducido por el desaparecido Julio Viteri Gamboa (Quito, 8 de enero 1908-Milagro, 28 de enero 1986), reconocido como coautor de esta ponencia por su dedicación incansable.

En el informe preliminar sobre el corte J, Estrada presenta su evaluación personal de lo logrado: «A la luz de los nuevos descubrimientos de la cultura Valdivia, y a los cuales nos referimos más adelante, se aprecia que el periodo A de nuestra cultura se inicia con cerámica cruda, con pocos elementos decorativos simplísimos, sobre un complejo cultural precerámico». (Estrada, 1961: 5)

Resultados que no llegaban a incorporarse cabalmente en la monografía posterior (Meggers et al., 1965: 17, 88, figs. 50-51; Bischof, 1979: 358, nota 73; Marcos, 1988, 1: 86).

Por encargo de los organizadores del Primer Simposio de Correlaciones Antropológicas AndinoMesoamericano realizado en Salinas, del 25 al 31 de julio de 1971 (Daggett, 1978), y con el deseo de reabrir la discusión sobre la estratigrafía temprana de Valdivia, Viteri volvió a excavar una sección del antiguo corte J, ampliándolo hacia el noreste (fig. 2). Invitó al autor presente, junto con la arqueóloga peruana Rosa Fung (Lima), a documentar la estratigrafía revelada en los perfiles del corte. Después del simposio, Viteri y el autor presente decidieron continuar el trabajo en forma mancomunada (Bischof \& Viteri, 1972). Ha llegado el tiempo de revaluar las evidencias recuperadas, con el fin de estimular nuevas investigaciones para resolver los interrogantes que aún subsisten.

Después de todo, estamos tratando de un paso constitutivo dentro del proceso de Neolitización forjado por las antiguas sociedades en la costa del Ecuador. Aunque haya alternativas más o menos precarias, la tecnología cerámica ofrece a una población sedentaria la mejor opción de procesar los alimentos vegetales producidos por una agricultura cada vez más desarrollada, lo que con más conveniencia se logra en vasijas resistentes al fuego. 
Entre Vegas y Valdivia: la fase San Pedro en el suroeste del Ecuador
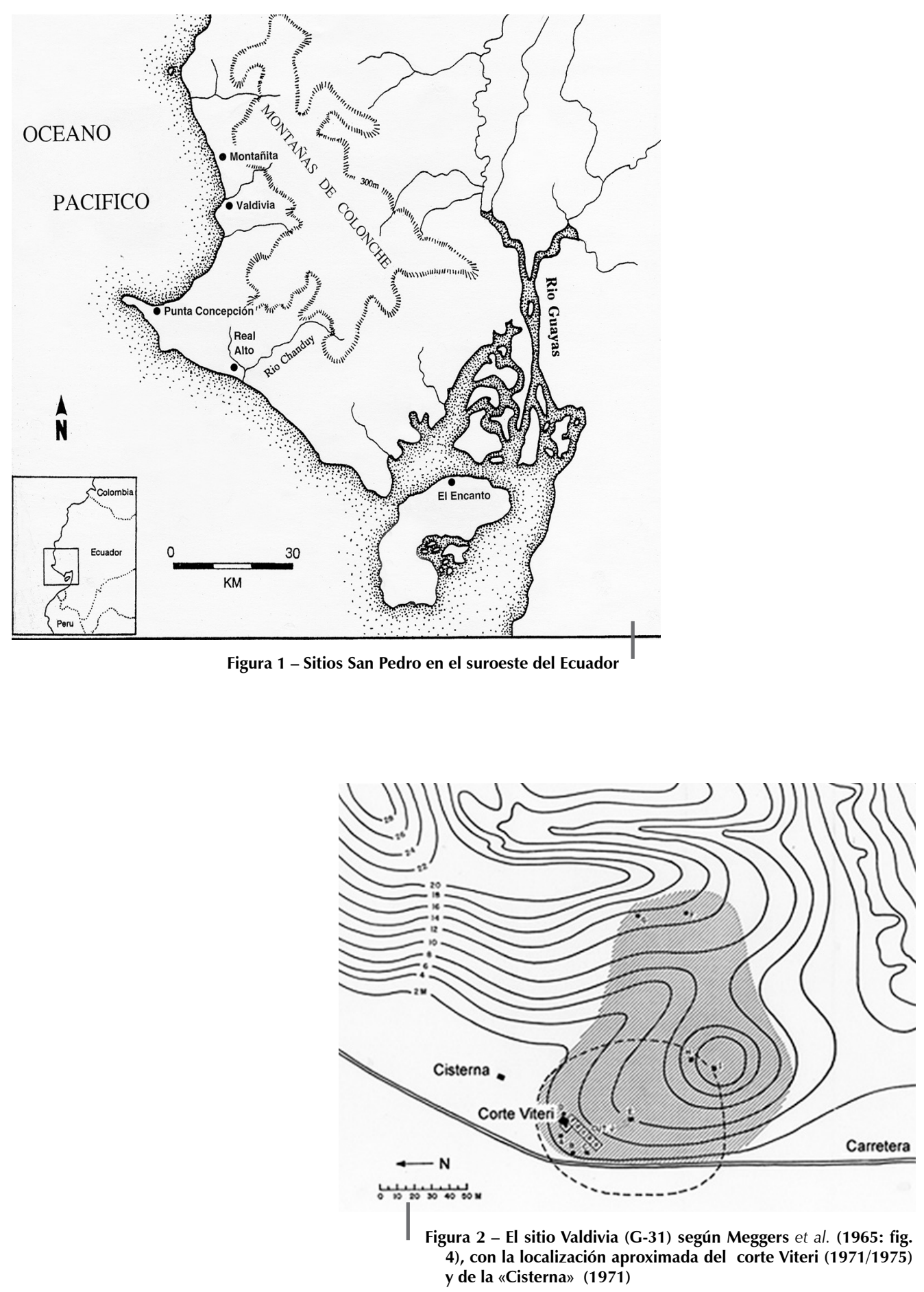


\section{LA FASE SAN PEDRO EN LA ESTRATIGRAFÍA DE VALDIVIA}

La excavación del Corte Viteri, sector E, trató de seguir en lo posible a los estratos físicos («naturales») que se destacaron en los perfiles ya preparados del corte (fig. 3). Debajo de un estrato superficial de la cultura Guangala, apareció el conocido basural cenizoso formado en su mayor parte durante los periodos 2-5 de la fase Valdivia (Hill, 1972-1974: lám. IV-VI; Bischof, 1979: fig. 16 a-h). El único elemento estratigráfico notable dentro de este basural fue una antigua grieta de erosión que bajaba en forma irregular, llevando en su sedimento de arena amarilla, numerosos artefactos Valdivia arrastrados, mayormente tiestos cerámicos, objetos líticos y restos de moluscos. Felizmente, la grieta no afectó a los estratos inferiores, ni al pozo o aplanamiento artificial que ocupaba el sector noreste del corte (unidad E 6), y cuyo relleno solo contenía fragmentos cerámicos del periodo Valdivia 1 (Bischof \& Viteri, 1972: fig. 1; Hill, 1972-1974: lám. II), fuera de dos fragmentos decorados de lo que después vino a denominarse cerámica San Pedro (Bischof, 1979: fig. 8 g-h)1. Este pozo o aplanamiento, a su vez cortaba la parte superior de una formación arenosa, acumulada a manera de playa o duna contra el pie noreste de un promontorio cercano a la desembocadura del río Valdivia que puede haber contribuido a su formación (estratos E 7-20).

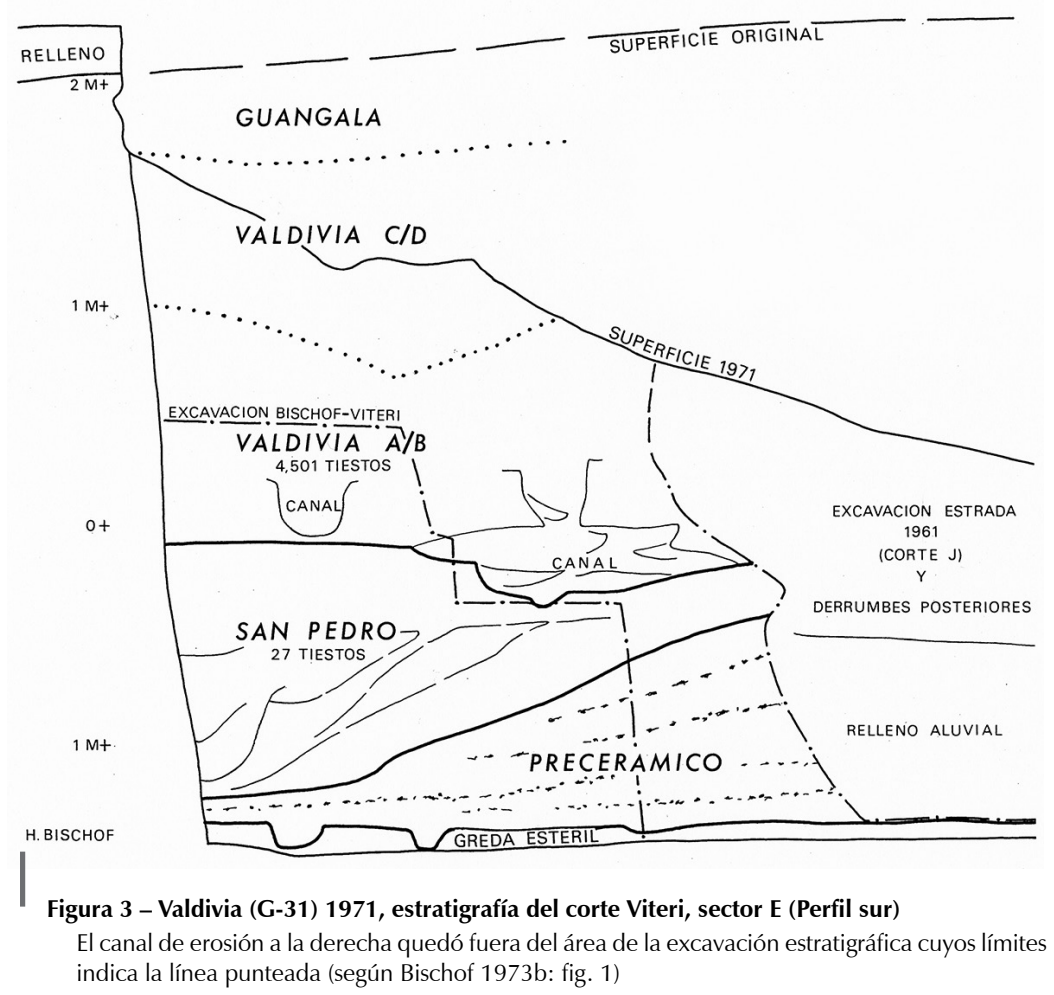

1 Esta asociación no es necesariamente significativa en términos culturales. Los tiestos pueden haber sido introducidos al rellenarse el pozo en la antigüedad o durante la excavación que hicimos, al raspar la pared y el fondo del pozo antiguo. 
Debajo de una capa amorfa de unos $15 \mathrm{~cm}$, interpretada como superficie disturbada por el tránsito peatonal o agencias naturales, se descubrieron una serie de 7-8 fogones superpuestos dentro de sedimentos arenosos que contenían abundantes piedras quemadas, trozos de carbón vegetal y restos de fauna marina, especialmente huesos de pescados relativamente pequeños. Al mismo tiempo, y de forma inesperada, se hallaron fragmentos de una cerámica desconocida, denominada San Pedro por los autores. Los fogones y hornos pavimentados, con las capas de arena intercaladas, alcanzaban la altura total de unos 1,40 m, señalando la presencia de un asentamiento de pescadores aún relativamente pequeño en los alrededores, núcleo de lo que vino a ser el pueblo de Valdivia posteriormente. De otra manera, esta zona de fogones podría haber sido un sitio de aprovisionamiento frecuentado por personas radicadas en zonas alejadas, tal vez tierra adentro, que se dedicaban a la pesca durante ciertas horas o temporadas.

Los deshechos culturales más antiguos se encuentran incrustados en el fondo natural de greda que se asemeja a la superficie de una zona pantanosa al margen de un manglar.

Esta misma formación estratigráfica había sido identificada por Julio Viteri en el año de 1960 durante su excavación del corte J, sectores d-f (Lyon, 1972-1974: 36-37), tratándose de la primera vez que se pudo definir un estrato físico con contenido cultural propio en el sitio de Valdivia. Una foto publicada por Meggers et al. (1965: lám. 9c) muestra a Viteri en el momento de enseñar a Emilio Estrada el lugar de un hallazgo cerámico especial, encima de un banco de tierra dejado sin excavar en el sector f. Según Viteri explicó al autor presente, este banco de tierra al mismo tiempo servía como testigo de la capa arenosa basal que tanto Estrada como él consideraban precerámica 2 - conclusión comprensible en vista de que los estratos precerámicos del corte Viteri aumentan hacia el antiguo corte J (Bischof, 1973b: fig. 1) -. Los estratos San Pedro de todas maneras no habrán arrojado un mayor número de tiestos cerámicos (Véliz, 1971).

La lámina 10b de Meggers et al. (1965) indica que la serie de fogones precerámicos y San Pedro registrados en el corte Viteri, continuaba en el corte J. Sin embargo, el análisis de los hallazgos a última hora se efectuó según el llamado «Método Ford» (Marcos, 1988, 1: 83-84), es decir en referencia a niveles métricos horizontales. Ya que estos no tomaron en cuenta el declive de los estratos físicos, se entremezclaron sus contenidos culturales. No sorprende entonces que los pocos fragmentos de cerámica San Pedro probablemente presentes en el corte J (d-f), no fueron reconocidos entre los abundantes tiestos Valdivia procedentes en realidad de los estratos fisicamente superpuestos (Meggers et al., 1965: figs. 50-51).

Mientras que los autores excavaban el corte Viteri (1971), algunos vecinos de Valdivia abrieron una trinchera de prospección unos $40 \mathrm{~m}$ hacia el noreste, según declararon «para un reservorio de agua» dentro de sus propios terrenos. Esta «cisterna» fue profesionalmente excavada, manteniéndose las paredes verticales hasta el piso estéril a los 2,10 m de profundidad. Se permitió al autor presente recoger varios lotes cerámicos superpuestos en su parte más baja, a los 2,10-1,40 m de profundidad, y sobre una superficie de 4,80 x 0,70 m. Los lotes recuperados sin selección alguna (Bischof, 1979: figs. 10-13, 14) sirvieron a Betsy Hill (1972-1974: 2-4) para confirmar su definición del periodo Valdivia 1. Merece destacar en esta ocasión que la secuencia Valdivia esbozada por Hill (1972-1974), no es un mero paráfrasis pormenorizado de la seriación anterior, como opinan Raymond et al. (1998: 160), sino que proyectó por primera vez una imagen nítida de los inicios y desarrollo temprano de la fase Valdivia así como de sus periodos tardíos. No se encontró ningún fragmento San Pedro en los lotes cerámicos de la «cisterna», así que puede suponerse que el yacimiento San Pedro no se habrá extendido a este sector. En lo que se refiere al periodo Valdivia 2, por otra parte, sus formas y decoraciones diagnósticas aparecieron por encima de las capas del Valdivia 1, a los 0,70 m sobre el piso natural (profundidad 1,00-1,40 m; Bischof, 1979: fig. 15).

2 El hecho de que Emilio Estrada reconociera este estrato precerámico basal de Valdivia, consta en su carta de instrucciones para Julio Viteri, escrita durante la excavación de los sectores e-f del corte J. Agradezco al Ing. Antonio Viteri una copia fotostática de esta carta conservada en su archivo personal. 
Una segunda excavación conjunta en Valdivia se realizó a principios de 1975, ampliándose el corte de 1971 hacia el sur (Bischof, 1979: fig. 5). El volumen de los deshechos culturales cernidos $\left(6 \mathrm{~m}^{3}\right)$ en este sector $(\mathrm{G})$ fue algo mayor que en el sector E estudiado en $1971\left(4,8 \mathrm{~m}^{3}\right)$, y salió un número correspondiente de tiestos San Pedro, exclusivamente - 30 en total-, algunos de los cuales podían ser pegados a los 27 fragmentos encontrados anteriormente (figs. 4-6).

En los últimos dos estratos encima del piso natural de greda, no se registraron hallazgos cerámicos esta vez tampoco. Queda así en pie la antigua propuesta de Estrada y Viteri, de alguna ocupación precerámica en Valdivia, aunque cabe admitir que la ausencia de cerámica podría ser fortuita en vista de su escasez en las capas superpuestas. Si este yacimiento formaba o no parte de la fase Las Vegas, solo podría determinarse en base a un estudio comparativo muy detallado de los artefactos líticos que aún no ha sido realizado. Entre los estratos pre- o acerámicos y aquellos que arrojaron cerámica San Pedro, no se observó ningún cambio en la utilización del espacio ni en el proceso de formación del mismo sedimento.

Si la densidad muy baja de unos 5-6 fragmentos cerámicos por metro cúbico cernido es representativa para otros sitios con cerámica San Pedro, su presencia será dificil de detectar en prospecciones superficiales y sondeos de otros sitios, pero de todos modos es factible gracias a la evidencia de Valdivia.

\section{SAN PEDRO Y EL PERIODO VALDIVIA 1: APRECIACIONES CERÁMICAS}

La pasta de la cerámica San Pedro es homogénea, densa y fina, con pocos granos de arena mayores, y relativamente dura. En las superficies cuidadosamente alisadas (San Pedro Alisado) y hasta pulidas (San Pedro Pulido), generalmente se notan las estrías finas de la herramienta empleada. El color marrón grisáceo más o menos oscuro de los cerámios se debe a la escasez del oxígeno al momento de la cocción. Este conjunto de rasgos técnicos distingue a la cerámica San Pedro del tipo Valdivia Inciso cuya decoración también es diferente según las muestras publicadas (Meggers et al., 1965: 63-65 y láminas correspondientes). En los estratos superiores San Pedro, algunos rasgos técnicos se acercan a las modalidades del Valdivia temprano: la pasta más arenosa/granulosa, líneas de pulimiento brillantes, y una cocción que admitió más oxígeno resultando en un color rojizo (fig. 4).

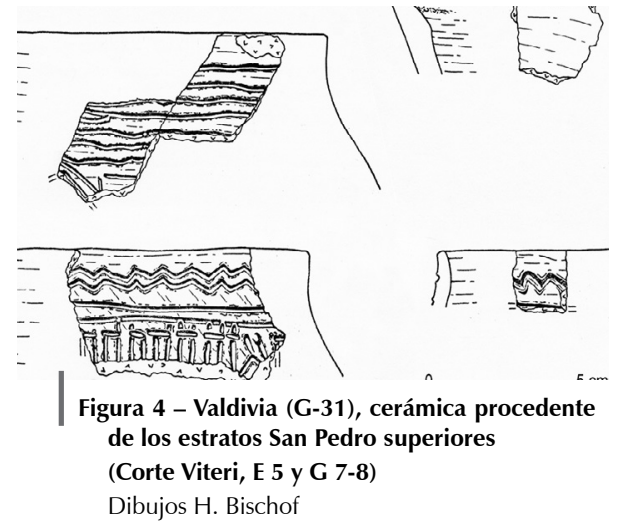

Aún en el material ampliado por la excavación del año 1975, la única forma cerámica documentada es una olla globular relativamente pequeña con el labio doblado hacia arriba, o cuello cilíndrico bajo ligeramente evertido a veces, similar a la forma 18 de Valdivia (Meggers et al., 1965: 92, figs. 54, 56). Del estrato San Pedro más alto procede el fragmento de un cuenco con borde biselado interior (fig. 4, derecha), parecido a la forma 2 del Valdivia Inciso Línea Ancha (Meggers et al., 1965: fig. 24; Marcos, 1988, 1: 232, $n^{\circ} 47$ ). Por falta de decoración, sin embargo, no puede confirmarse si formaba parte del conjunto cerámico San Pedro.

Según parece, el ajuar cerámico San Pedro presente en Valdivia, consiste de una sola clase de ollas. Ya que los alimentos generalmente se prepararon al fuego abierto, o por medio de piedras calentadas, la rareza de las vasijas cerámicas y su tamaño pequeño indican que cumplieron alguna función importante pero especial. Esto lo confirma el porcentaje muy alto de tiestos decorados - 

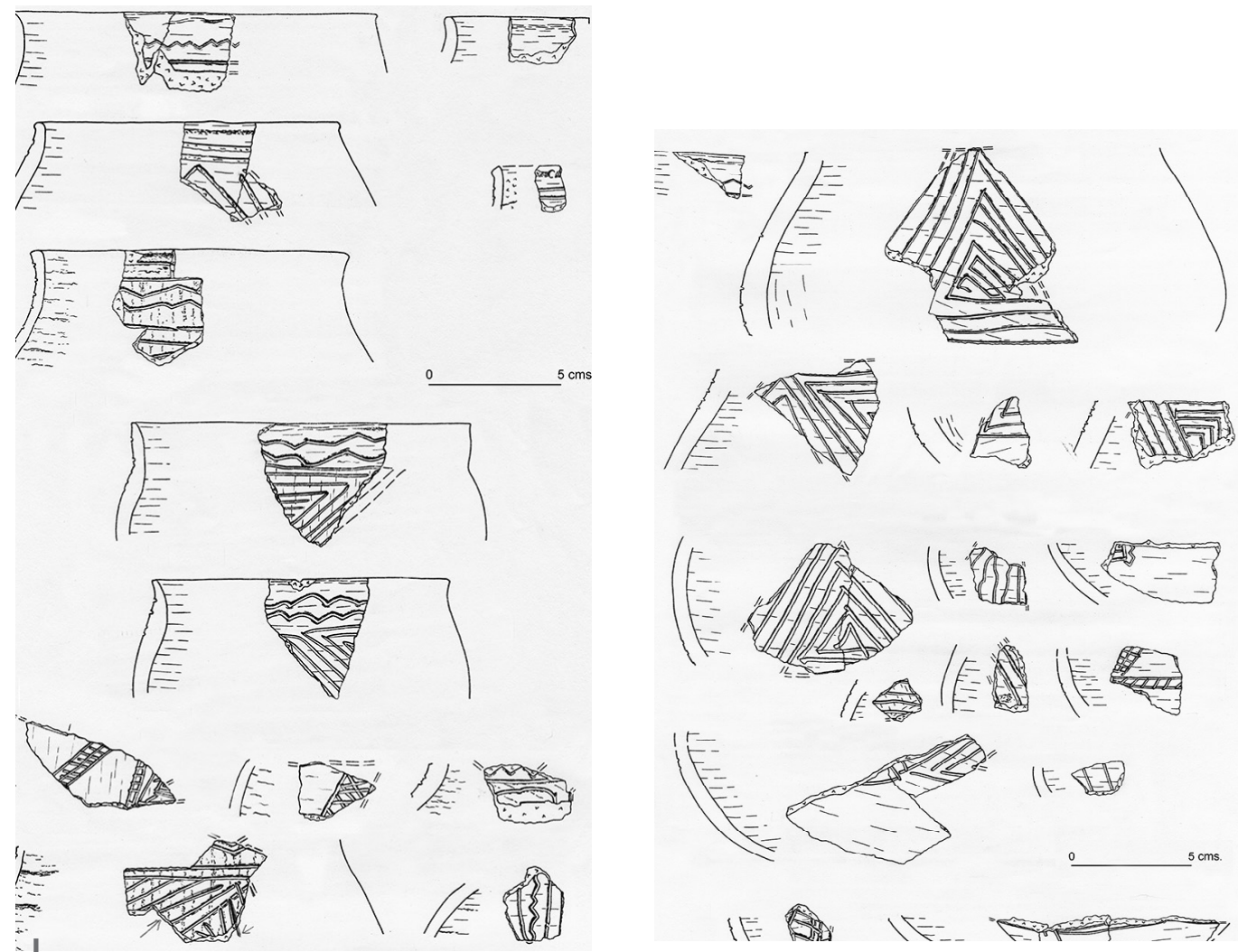

Figuras 5 y 6 - Valdivia (G-31), cerámica procedente de los estratos San Pedro medios e inferiores (Corte Viteri, E 7-18, G 12-19)

Dibujos H. Bischof

$67,5 \%$ - según el cual, casi todas las superficies estaban decoradas por su mayor parte. Son pertinentes las observaciones de Augusto Oyuela acerca de las funciones sociales de la cerámica, aparte de las «utilitarias» (Raymond et al., 1998: 166-167). En este sentido, la cerámica San Pedro proporciona un ejemplo aún mejor que San Jacinto (Colombia) donde solamente un 20$30 \%$ de los tiestos llevan decoración.

Como queda notado, el estilo de decoración San Pedro difiere de los tipos Valdivia Inciso/Inciso Línea Fina (Meggers et al., 1965: láms. 61-64 y otras), a pesar de que puede repetirse tal cual componente particular de los motivos, como las fajas con hachurado cruzado3. Los diseños se trazaron en forma muy precisa por líneas incisas, o más bien cortadas en la arcilla húmeda. La mayoría de los motivos se componen de líneas rectas equidistantes. A veces forman diseños complejos que no son simétricos y pueden incorporar áreas llenadas por meandros simples. En varios casos, una serie de triángulos concéntricos alternados cubre toda la parte superior de la

3 El fragmento decorado con rectángulos cuadriculados (Meggers et al., 1965: lám. 183f), originalmente considerado como posible muestra de la cerámica San Pedro, probablemente forma parte del conjunto Valdivia 1 ó 2, según un hallazgo gemelo en Real Alto (Damp, 1979: lám. 84a). Meggers (1987: 27) hace notar por otra parte que los otros dos fragmentos mencionados en un principio (Bischof \& Viteri, 1972: 549), encajan dentro de las normas del Valdivia Inciso (Meggers et al., 1965: 64j, q). 
vasija. Como elementos especiales deben nombrarse el hachurado en zonas, simple o cruzado, así como las diagnósticas líneas zig-zag que rodean al borde.

El contraste muy marcado entre la cerámica San Pedro y aquella del Valdivia 1, estratigráficamente superpuesta en el corte Viteri, unidad E 6 (Bischof \& Viteri, 1972: fig. 1; Hill, 1972-1974: lám. II; Bischof, 1979: fig. 9), indica que el hiato estratigráfico observado era significativo también en términos culturales.

En primer lugar, se observa una especialización técnica-funcional de la cerámica Valdivia 1 que constituye un gran avance en referencia a lo que se conoce de la cerámica San Pedro. Ahora, las vasijas destinadas a cocinar, transportar y distribuir líquidos, se distinguen claramente de aquellas para el uso personal, en términos tecnológicos así como por su forma y decoración (DeBoer, 2003): ollas y cuencos grandes sin decoración de los tipos Pozo Ordinario, Pozo Pulido en Líneas, y Pozo Pulido (Bischof, 1979: 356-359); ollas con decoración peinada o en algunos casos, decoradas con incisiones más o menos cuidadosas; cuencos para el servicio personal más finamente elaborados, con decoración rasguñada. Hay otras diferencias como el acabado de las superficies y la presencia de un engobe rojo, aunque a veces mal logrado, que por primera vez se emplea en forma sistemática en el Valdivia 1. Incluso empezaron en aprovecharse ya en este periodo temprano hasta las propiedades tecnológicas de las diferentes pastas cerámicas para usos distintos (Marcos, 2003: 21). La cantidad impresionante de tiestos presentes en cualquier contexto Valdivia señala que ahora, sí, la cerámica formaba parte integral de la vida cotidiana y ceremonial.

De todo esto se desprende que la separación terminológica entre la fase San Pedro por un lado, y Valdivia por el otro, no es arbitraria, a pesar de que se noten ciertos elementos de continuidad entre ambas tradiciones alfareras. Esta se expresa, por ejemplo, en las pequeñas ollas que guardan una relación estrecha con sus antecedentes San Pedro (Bischof, 1979: fig. 8c: San Pedro, y 10d: Valdivia 1). Su decoración incisa, sin embargo, en el Valdivia 1 se traza en forma irregular (Hill, 1972-1974: 4). Una relación directa se manifiesta también en la decoración incisa con esmero, relativamente rara en el Valdivia 1 de Valdivia (Bischof, 1979: fig. 14 I-m, op). Lo mismo se observa en referencia a los motivos «rasguñados» del Valdivia Inciso Línea Fina temprano. Por otro lado, el fragmento de un cuenco con decoración San Pedro procedente de Montañita, similar a una forma Valdivia encontrada en la «cisterna» (Bischof, 1979: fig. 12c), en términos tecnológicos se parece mucho a las pequeñas ollas del estrato San Pedro más alto de Valdivia (Bischof, 1979: fig. 8c). Según este hallazgo, el desarrollo de formas específicas para determinadas funciones se habrá iniciado ya en los tiempos tardíos de la fase San Pedro -en este caso, imitando cuencos cortados de un mate-. En OGSE-80, sitio tipo de la cultura Las Vegas, efectivamente se registró la presencia de Lagenaria siceraria en la costa del Guayas desde épocas muy remotas (Stothert, 1988: 215-216).

La validez de la fase San Pedro después de todo eso, no depende solamente de 57 tiestos seleccionados sin contexto, impresión errónea sugerida por varios comentaristas (p. e. Ledergerber, 1983: 29, 30; Schwarz \& Raymond, 1996: 208, nota 2; Raymond et al., 1998: 160). Más bien, se basa en una formación estratigráfica que excede los 1,40 m en profundidad y que atestigua actividades sostenidas vinculadas con la preparación de alimentos, mayormente de origen marino. Un hiato cultural se nota en referencia al Valdivia 1, periodo que separó a su vez, la fase San Pedro del Valdivia plenamente desarrollado (Periodos 2-5) con su gran variedad de técnicas decorativas, entre ellas el llamado «Valdivia Inciso». Si la calidad de la cerámica San Pedro es superior a los fragmentos supuestamente contemporáneos de Achallán en la Península de Santa Elena (Stothert, 1976: 91-93; 1988: 232-233), esto se debería tal vez a su mayor cercanía a la región donde se habría desarrollado la tecnología cerámica en la costa del Ecuador4.

4 Según Stothert (1988: 232), la cerámica encontrada en Achallán en conjunto «llenaría una cucharilla de té». Por su pasta arenosa, podría tratarse de tiestos Valdivia fuertemente erosionados, posibilidad que ya fue considerada por Karen Stothert (1976: 91). 


\section{3. ÁREA DE DIFUSIÓN CONOCIDA. ESTRATIGRAFÍAS COMPARADAS}

No se ha realizado una búsqueda sistemática de asentamientos San Pedro en los años transcurridos desde su primer descubrimiento. Sin embargo, fragmentos cerámicos con decoración tipo San Pedro —o parecida a ella— han sido detectados en cuatro lugares más: El Encanto (Puná), Punta Concepción, Real Alto, y Montañita (fig. 1).

Los datos estratigráficos de El Encanto, isla de Puná, corresponden en parte a los que se dieron en Valdivia: dos de los siete fragmentos se encontraron directamente superpuestos a un asentamiento precerámico tardío (Porras, 1973: 155-159, lám. 30e, h; Damp \& Vargas, 1995: 159-60). Es difícil saber hasta qué punto agentes naturales o antropógenos, e incluso el método de excavación métrica, afectaron el contexto estratigráfico de los demás fragmentos cuya atribución al San Pedro merece comprobarse.

Entre los hallazgos de Punta Concepción en la península de Santa Elena, sitio tipo del Valdivia 1 (Hill, 1972-1974: 2-3), figura por lo menos un fragmento tipo San Pedro, según los dibujos que la desaparecida investigadora Betsy Hill hiciera accesibles oportunamente al autor presente. El sitio es notable por la ausencia de hallazgos Valdivia 2. Por otra parte, los tiestos con decoración incisa, más frecuentes aquí que en el material Valdivia 1 de Valdivia, señalarían una continuación de las tradiciones San Pedro. J. Scott Raymond pudo demostrar que la planta de la aldea tenía forma de herradura, por los restos de moluscos concentrados alrededor de las supuestas casas (Marcos, 1988, 1: 86). Sin embargo, la capa cultural muy superficial no permitió establecer subdivisiones estratigráficas, ni tampoco parecen haberse recuperado contextos habitacionales tales como pisos de vivienda, pozos o fogones.

En Real Alto (excavación J. Damp) se encontraron cinco tiestos con decoración San Pedro ubicados estratigráficamente entre el Valdivia 1 y el Valdivia 2 (tardío), según opinan los excavadores en base a las respectivas profundidades métricas (Damp, 1979: fig. 3; 1988: fig. 2; Damp \& Vargas, 1995: 161, fig. 13.4). Sin embargo, la distribución de los tiestos San Pedro en una profundidad uniforme parece señalar un antiguo nivel de superficie. Por otra parte, Damp (1988: fig. 4) informa sobre los numerosos pozos y trincheras de cimentación que ocurren en esta parte de Real Alto, difíciles de observar bajo las condiciones locales. El mismo reconoce que «la mezcla muy extensa de los restos desechados en realidad impide en un basural tan profundo como aquel de Real Alto, de evaluar y controlar la clasificación [Valdivia] de Norton» (Damp, 1979: 90; traducción H. B.).

El material más profundo del Valdivia 1, en estas condiciones bien puede proceder de unos pozos que perforaron la antigua superficie sobre la cual yacían los tiestos San Pedro. Me refiero especialmente a las numerosas «apachitas» o «túmulos» (Damp, 1988: 69-71) que más bien deben ser interpretadas, igual que los famosos «cairns» de Loma Alta, como material lítico depositado en el fondo de algunos pozos de almacenamiento cuyos límites superiores no se lograron de observar (Marcos, 1986: 31-32; Marcos \& Michczynski, 1996: 97).

Finalmente, en el sitio de Montañita, Provincia del Guayas, redescubierto en el año de 2005, una búsqueda superficial arrojó por lo menos un fragmento San Pedro entre abundantes materiales del Valdivia 1 y 2, disturbados por el corte del basural practicado durante la construcción del oleoducto de Manta, kilómetro 59, y la actividad de excavadores clandestinos posteriores.

Evidentemente se trata de un centro importante situado unos $15 \mathrm{~km}$ al norte de Valdivia. Sus restos visibles cubren unos $150 \times 80 \mathrm{~m}$ encima de una montaña aplanada que forma parte de una terraza pleistocénica, unos $30 \mathrm{~m}$ sobre el fondo fertil de un pequeño valle. El suelo cenizoso típico de un basural Valdivia se extiende sobre la parte superior de la falda sur fuertemente empinada, alcanzando una profundidad de 1,30 m. Montañita ocupa una posición ecológica privilegiada similar a la de Valdivia, la que permite un acceso fácil tanto a los recursos marítimos como a los terrestres, ya que el cauce principal del río dista unos $650 \mathrm{~m}$ hacia el sur mientras que la playa actual del mar solo dista unos 300 m en dirección oeste. 
En todos los cuatro sitios, los tiestos fueron seleccionados de lotes cerámicos más amplios, por su decoración parecida al San Pedro; solo Porras (1973: 159) se refiere también a los rasgos tecnológicos. Dada la baja frecuencia de hallazgos cerámicos en los estratos San Pedro de Valdivia, no sorprende el número reducido de tiestos identificados en cada uno de los sitios. Según ellos, el área de difusión de la cerámica San Pedro es de marcada orientación marina. Cubre la parte sur del área cultural Valdivia, ceñiéndose a la costa oeste de la provincia del Guayas.

\section{1. Elementos de cronología relativa en el sitio de Valdivia}

Todos los sedimentos arenosos encima del antiguo fondo de manglar, se acumularon durante los periodos precerámicos y San Pedro, posiblemente debido a la tala del manglar y destrucción de la vegetación sobre la colina colindante causada por la presencia del hombre. Cabe destacar que dentro de esta formación que llegó a alcanzar unos 1,70 m de espesor en el corte J, ningún hallazgo señala una ocupación contemporánea —o antecedente- del Valdivia 1 cuestas arriba. La propuesta de Damp \& Vargas (1995: 162) a tal efecto probablemente fue causada por la localización errónea de la «cisterna» en el mapa publicado por el autor presente (Bischof, 1979: fig. 4)5. El basural Valdivia 1 en realidad se extiende en la parte baja hacia el noreste, de donde ningunos materiales pueden haber sido arrastrados por agencias naturales, hacia el área del corte Viteri sobre las estribaciones del promontorio (fig. 2).

No hay evidencia intrínseca cultural o radiométrica que permita calcular el tiempo transcurrido durante la acumulación de los estratos San Pedro: puede tratarse de pocos años o hasta de varios decenios. La capa amorfa encima de ellos, formada cuando cesaron las actividades culinarias en este sector, sin embargo indica cierto lapso de tiempo transcurrido.

El lote cerámico Valdivia 1 recuperado por encima de los estratos San Pedro procede de un corte practicado en la superficie de los mismos, sea en forma casual, como camino erosionado, o sea intencional, como aplanamiento para una terraza o casa. No se trata de una grieta de erosión como lo imaginan Damp \& Vargas (1995: 162), pero su relleno de igual manera puede haber sido redepositado ya que los fragmentos cerámicos encontrados en el mismo, son pequeños y proceden de muchas vasijas diferentes.

Según los datos actuales, los basurales del Valdivia 1 se concentran en el fondo de la ensenada formada por el mismo promontorio y las colinas colindantes hacia el este. Muchos tiestos recuperados de la «cisterna» abierta en esta zona relativamente abrigada, representan una gran parte de las respectivas vasijas, lo que demuestra que esta vez, sí, se trata de basura primaria. Consta en la «cisterna», igual como en el corte Viteri, la superposición del Valdivia 2 sobre el Valdivia 1 -continuidad estratigráfica y tipológica evidente que difícilmente admite una fase San Pedro entrecalada entre los dos-.

Las hipótesis de Damp \& Vargas (1995: 162) sobre la secuencia cultural, y la forma del asentamiento durante las épocas tempranas, no son convincentes porque pasan por alto la topografía local de Valdivia. Esta se impone tan fuertemente que las comparaciones con sitios localizados en áreas planas como Loma Alta o Real Alto, son muy poco iluminantes.

\section{CRONOLOGÍA RADIOMÉTRICA}

La secuencia de las fases o periodos Precerámico (¿Las Vegas?)/Acerámico $>$ San Pedro $>$ Valdivia $1>$ Valdivia $2>$ Valdivia 3 y posteriores, queda demostrada por superposición estratigráfica en el corte Viteri. Una nota discordante introducen las mediciones del radiocarbono que se refieren al estrato pre- o acerámico en el fondo de la estratigrafía de Valdivia, porque a primera vista parecen ser muy recientes frente a la antigüedad del Valdivia 1(a) vislumbrada por otros autores.

5 El sondeo D de Emilio Estrada, apenas mencionado por Evans et al. (1959: 15), tampoco parece ser ubicado correctamente en relación al corte J (Meggers et al., 1965: 16 y fig. 4). 
En eso, sin embargo, hay algo de relativo ya que John W. Hoopes (1994: 18-19) con buenas razones puso en tela de juicio precisamente los ensayos más antiguos GX-5269, ISGS-448 y GX-5267. A pesar de eso, siguen encabezando la lista de James A. Zeidler (2003: 509, tabla A 1) quien propone en base a ellos, una antigüedad de 4400-3800 calA.C. para un sub-periodo «Valdivia 1a», y 3800-3300 calA.C. para un sub-periodo «Valdivia 1b» (Zeidler, 2003: 519, tabla A 2). Las cifras para el periodo Valdivia 1 en conjunto oscilan entre 5400-4850 calA.C. (GX5269) y 3340-2920 calA.C. (L-1042C; Zeidler, 2003: tabla A 1), hecho que debería despertar dudas y en todo caso, requiere explicación. Aun muestras bien documentadas procedentes de contextos antropógenos, al parecer no siempre valen como hitos de la historia cultural.

Es desconocido si hay antecedentes de los materiales Valdivia 1 registrados en Valdivia (Bischof, 1979) que justificarían la antigüedad superior que se les atribuye a los hallazgos tempranos de Real Alto y Loma Alta. En lo que se refiere al periodo Valdivia 1, sus subdivisiones Valdivia 1a y $1 \mathrm{~b}$ nunca han sido demostradas por medio de hallazgos y contextos arqueológicos. Fuera de la evidencia de Valdivia publicada por el autor presente (Bischof, 1979), ni siquiera existen pruebas para poder definir el Valdivia 1 y 2 como periodos separados, ya que el manuscrito de Betsy Hill sobre Punta Concepción quedó sin publicar. El cansancio de realizar y publicar esta clase de estudios básicos (Damp, 1988: 25; Damp \& Vargas, 1995: 157), en estas condiciones es prematuro y por lo demás, mal apropiado en cualquier campo de investigación científica, sea como fuera su respectiva orientación teórica.

Se disculpará al autor presente de interpretar los datos en forma diferente, dando prioridad a la estratigrafía observada, y fácil de comprobarse en Valdivia. Seis de los siete ensayos que se refieren al estrato más profundo (E 20) de la formación precerámica/San Pedro, basados en muestras de carbón vegetal, dieron fechas entre 3650-3370 calA.C. y 3360-2930 calA.C., sin tomarse en cuenta el ensayo Hv-4675 que resultó más reciente por razones desconocidas6. El mismo lapso de tiempo está indicado por las muestras procedentes de las capas correspondientes del corte J de Estrada (Meggers et al., 1965: 149, Tabla G). Según estos datos, la ocupación precerámica/ San Pedro más antigua de Valdivia ocupará algún lapso de tiempo entre 3600 y 3350 calA.C. Ya que los estratos San Pedro parecen haberse formado en un tiempo relativamente corto, los comienzos del Valdivia 1 bien podría remontarse hacia 3550-3500 calA.C.

6 Agradezco al Dr. Mebus Geyh, Jefe del $~^{14} \mathrm{C}$ - und ${ }^{3} \mathrm{H}$-Labor des Niedersächsischen Landesamts für Bodenforschung» (Hannover, Alemania), la elaboración de los siguientes ensayos (Bischof, 1973a: 270-273), los que fueron calibrados amablemente por su sucesor Prof. Dr. Manfred Frechen, jefe del Departamento Científico de lo que hoy viene a ser el «Leibniz-Institut für Angewandte Geowissenschaften» (Hannover), en comunicación personal del 7 de julio 2006:

código

$\mathrm{Hv}-4673$

años a.p. cal. (probabilidad 68,2\%) proveniencia

$4335 \pm 100 \quad 3305-2760$ a.C. Loma Alta, J 160

Hv-4837 $4095 \pm 90 \quad 2860-2495$ a.C. Valdivia, Viteri, E 3

Hv-4838 $4260 \pm 100 \quad 3015-2665$ a.C. Valdivia, Viteri, E 4

Hv-4674 $4510 \pm 95 \quad 3355-3040$ a.C. Valdivia, Viteri, E 20

Hv-4675

Hv-4839

$4075+110$

$4535 \pm 55$
"

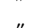

Hv-4840 $4495 \pm 140 \quad 3360$ - 2930 a.C. " " Precerámico

Otra muestra elaborada por la Dra. Marianne Münnich, entonces Directora del Laboratorio ${ }^{14} \mathrm{C}$ del «Institut für Umweltphysik der Universität Heidelberg» (Alemania), fue calibrada por el Dr. Bernd Kromer de la misma institución (com. pers., 16 de agosto 2006):

código años a.p. cal. (probabilidad. 68,2\%) proveniencia contexto

Hd-3810 $\quad 4760 \pm 80 \quad 3640$ - 3510 a.C. Valdivia, Viteri, E $20 \quad$ Precerámico

Nota: el rango indicado tiene una probabilidad del 53,4 \%; otro rango para el mismo ensayo, es de 3430-3380 a.C., con una probabilidad del $14,8 \%$.

Dos mediciones fueron efectuadas por el Illinois State Geological Survey, gracias a la oportuna intervención de Donald W. Lathrap (Ziólkowski et al.,1994: 147; calibradas según Zeidler, 2003: 510, tabla A1):

código años a.p. cal. (probabilidad. 68,2\%) proveniencia contexto

ISGS-275 $4700 \pm 80 \quad 3630-3370$ a.C. Valdivia, Viteri, E $20 \quad$ Precerámico

ISGS-274 $4580 \pm 80 \quad 3500-3100$ a.C. " $\quad$ Precerámico 
La mayor parte de los datos presentados por Marcos \& Michczynski (1996: 98, tabla 1 y fig. 1a) está compatible con este reordenamiento. Como consecuencia positiva, la duración del periodo Valdivia 1 se reduce de los 1100 años calculados por Zeidler (2003: 319) a unos 300-400 años es decir, 3500-3200 calA.C., aproximadamente. Con sus márgenes de error impresionantes, las dataciones por termoluminescencia admiten la «cronología corta» aquí propuesta, la que implica al mismo tiempo un ritmo más acelerado de las innovaciones (cerámicas) en el Formativo de la costa del Guayas (Marcos \& Michczynski, 1996: 111, apéndice 2).

Los mismos autores (Marcos \& Michczynski, 1996: 97) no dejan de identificar la causa probable de la discrepancia entre expectativas y resultados recibidos:

«Por otra parte, el reuso y la costumbre de reemplazar los postes de las paredes podrían indicar que un buen porcentaje de la madera quemada en los fogones y hornos instalados dentro de pozos, consistió de antiguos materiales de construcción desechados. Maderas resistentes como el algarrobo y guasango proporcionarían una fecha varios siglos más antigua correspondiente al tiempo cuando se cortó el arbol y no cuando acabó de ser usado como leña» (traducción H. B.).

Peor todavía, el carbón procedente de un tronco quemado en realidad informa sobre algún episodio de su crecimiento, lo que facilmente puede añadir siglos al resultado de la datación. Muchos ensayos fecharán entonces la antigüedad de la vegetación talada en vez del contexto cultural asociado. Tal vez no sea una coincidencia que el fenómeno de las dataciones discordantes, y especialmente aquellas de antigüedad excesiva, se presente con mayor agudez durante el Valdivia 1 cuando se talaba el bosque primario para crear campos de cultivo.

Un detalle relevante al tiempo de comparar fechas, es el hecho que el carbón utilizado para las dataciones procedentes del corte Viteri, en gran parte procede de ramos, al contrario de lo que se ha sugerido para las muestras de Real Alto y Loma Alta. Muchos ramos al parecer sirvieron como brochetas para tostar las enormes cantidades de pescados pequeños cuyos huesos dan una matiz marrón a los estratos de basura precerámica/San Pedro. Esta práctica culinaria resultó en una combustión incompleta de la madera, explicándose así la abundancia de trozos carbonizados (Bischof \& Viteri, 1972: 551, fig. 1), en contraste con las capas Valdivia del sitio tipo y en otros sitios.

El segundo método principal de la preparación de alimentos en un ambiente aún largamente precerámico, es por medio de piedras calentadas, y también es más factible con ramas en vez de troncos. Son abundantes las piedras quemadas de tamaño mediano, fracturadas en forma angular, que por su mayor parte procederán de fogones pavimentados similares a los que fueron observados también en otros sitios fuera de Valdivia (Stothert, 1988: 90). En todo caso, las muestras de carbón vegetal del corte Viteri, representan los eventos culturales por fecharse en forma bastante directa, lo que no es tan cierto en Real Alto ni en Loma Alta como se ha visto. Para llegar a una cronología confiable de los inicios de la fase Valdivia, se necesitan más datos de estratigrafía comparada y un control aún mejor de las muestras usadas para las dataciones.

\section{CONCLUSIONES}

En la base norte de un promontorio cercano a la desembocadura del río Valdivia, alrededor de 3600 calA.C. Ilegó a formarse una zona de sedimentos naturales entremezclados con desperdicios humanos. Ella se extiende sobre un área mínima de $15 \times 8 \mathrm{~m}$, alcanzando por lo menos 1,60 m de grosor en su centro situado en el sector $f$ del antiguo corte $J$ de Emilio Estrada. Este área fue intensamente utilizada para la preparación de alimentos, tal vez por los ocupantes de una, o más probablemente varias casas familiares. Si bien faltan datos específicos sobre su contexto social (Raymond et al., 1998: 160), de todas formas se habrá tratado de un grupo sedentario que llevaba una vida de pescadores, siquiera por temporadas. Lo demuestran los abundantes restos de la fauna marina entre los cuales predominan los huesos de pescados relativamente pequeños. 
La misma preferencia de especies pequeñas como la anchoveta y la sardina, ha sido observada en sitios del Arcáico peruano (Shady, 2003: 103). Este tipo de pesca no es eficiente con anzuelos sino que requiere redes flotantes que en el Perú, desde el 4to milenio A.C. se tejieron de algodón, cultígeno cuya presencia no puede confirmarse tan tempranamente en el Ecuador, sin embargo (Marcos, 1998: 296-297). En vista de la cantidad de restos vegetales carbonizados preservados en el corte Viteri, es de lamentar que se perdieron las muestras orgánicas y del suelo guardadas en la antigua casa de Presley Norton al inundarse el barrio de Urdesa (Guayaquil), siniestro que también afectó las colecciones de otros investigadores.

Volviendo a la cerámica San Pedro encontrada en este contexto estratigráfico, la pista dada hace unos 35 años no ha sido perseguida por varias razones, entre las que figura el entusiasmo despertado por el descubrimiento de los horticultores del Formativo temprano en la costa del Ecuador (Raymond, 1999; 2003).

Aún si no todas las interrogantes han sido resueltas, los datos actuales permiten sostener que la cerámica San Pedro, fue un antecedente del Valdivia 1. Hasta la fecha nada indica de que se haya desarrollado localmente en ninguno de los cinco sitios donde fue encontrada —Montañita, Valdivia, Real Alto, Punta Concepción y El Encanto- de manera que su región de origen queda por ubicarse. Ya que la cerámica San Pedro no apareció en ninguno de los sitios estudiados por los proyectos conducidos en el interior del valle de Valdivia (Raymond, 1999: 157, nota 3), pero saltó a la vista en una visita casual al sitio de Montañita, su filiación con el ambiente litoral es evidente. Efectivamente, Raymond (1999: 153) deplora la ausencia de datos sobre las poblaciones de la faja costeña durante el Formativo temprano y en la época precerámica antecedente.

En resumidas cuentas, los orígenes de la cerámica en la costa del Ecuador están todavía sin elucidar a los cincuenta años después del descubrimiento de la cultura Valdivia. Ninguna hipótesis trata del origen del periodo Valdivia 1, con la excepción de lo que sugiere el descubrimiento de Altomayo (Damp \& Vargas, 1995: 165-166)7. Los argumentos esbozados en el trabajo presente, favorecen su desarrollo a partir de una base San Pedro.

En lo que se refiere a esta fase San Pedro, Betty J. Meggers (1987: 27-28, fig. 15) se empeñó en demostrar la similitud de sus motivos incisos con la decoración cerámica de tres sitios del Jomon Temprano tardío, o Jomon Medio temprano, en la bahía de Tokyo, isla de Honshu. Sin considerar probabilidades, su sugerencia de que no debería excluirse sin más estudio la posibilidad de que existan antecedentes Jomon de la cerámica San Pedro, quedará en pie hasta que una alternativa haya sido demostrada científicamente.

Finalmente, una nota acerca de la rareza de objetos cerámicos en la primera fase de su introducción a una sociedad precerámica, hecho que en el caso de San Pedro ha confundido a tantos autores. En La Galgada, departamento de Ancash (Perú), se encontró una sola vasija cerámica dentro de un sitio ceremonial esencialmente precerámico (Grieder et al., 1988: figs. 158-159). La vasija, similar a los mates tallados de Huaca Prieta por su forma, tamaño y el hecho de estar decorada, habrá cumplido funciones rituales análogas (Bischof, 1999: 107). De paso demuestra que al lado de la hipótesis del origen «casero» de la cerámica (Raymond et al., 1998: 167-168), de ninguna manera puede descartarse su origen dentro de un contexto ritual, seguido por la transmisión de objetos o conocimientos cerámicos de una sociedad a la otra. Solo cuando la mayor parte de la población llegaba a reconocer las ventajas y posibilidades de la nueva tecnología, comenzarían a producirse vasijas en grandes cantidades y con funciones diversas, como lo demuestra el ejemplo del conjunto cerámico Valdivia 1.

7 Los trozos de barro quemado en la casa precerámica de Altomayo cerca de Real Alto, atribuida a una fase «Chuculunduy» concebida para cubrir el espacio de tiempo entre Las Vegas y Valdivia (4650-3500 calA.C.), no se relacionan con ninguna tradición alfarera. Sin embargo, las figurinas simples de piedra asociadas hacen recordar aquellas del Valdivia 1. 


\section{Referencias citadas}

BISCHOF, H., 1973a - The Origins of Pottery in South America. Recent Radiocarbon Dates from Southwest Ecuador. In: 40 Congresso Internazionale degli Americanisti, 1: 269-281; Genova: Casa Editrice Tilgher. Roma-Genova 3-10 Settembre 1972, Atti.

BISCHOF, H., 1973b - Una investigación estratigráfica en Valdivia (Ecuador): Primeros resultados. Indiana, 1: 157-167.

BISCHOF, H., 1979 - San Pedro und Valdivia: Frühe Keramikkomplexe an der Küste SüdwestEkuadors. Ein Beitrag zur Kenntnis des südamerikanischen Formativums. Beiträge zur Allgemeinen und Vergleichenden Archäologie, 1: 335-389.

BISCHOF, H., 1999 - Los mates tallados de Huaca Prieta: ¿Evidencias del arte Valdivia en el Arcáico Centroandino? In: El Periodo Arcáico en el Perú: Hacia una definición de los orígenes (Peter Kaulicke, ed.): 85-119; Lima: PUCP. Boletín de Arqueología PUCP, 3.

BISCHOF, H. \& VITERI G., J., 1972 - Pre-Valdivia Occupations on the Southwest Coast of Ecuador. American Antiquity, 37 (4): 548-551.

DAGGETT, R. E., 1978 - The Life Cycle of an Idea: Transpacific Voyages and American Archaeology. Journal of the Virgin Islands Archaeological Society, 6: 13-22.

DAMP, J. E., 1979 - Better Homes and Gardens: The Life and Death of the Early Valdivia Community. Tesis doctoral, Department of Archaeology, University of Calgary. 164 p.

DAMP, J. E., 1988 - La primera ocupación Valdivia de Real Alto. Patrones económicos, arquitectónicos e ideológicos, 148 p.; Guayaquil: ESPOL-Quito: Corporación Editora Nacional. Biblioteca Ecuatoriana de Arqueología, t. 3.

DAMP, J. E. \& VARGAS S., L. P., 1995 - The Many Contexts of Early Valdivia Ceramics. In: The Emergence of Pottery. Technology and Innovation in Ancient Societies (William K. Barnett \& John W. Hoopes, eds.): 157-168; Washington-London: Smithsonian Institution Press. Smithsonian Series in Archaeological Inquiry.

DEBOER, W. R., 2003 - Ceramic Assemblage Variability in the Formative of Ecuador and Peru. In: Archaeology of Formative Ecuador. A Symposium at Dumbarton Oaks, 7 y 8 de octubre 1995 (J. Scott Raymond \& Richard L. Burger, eds.): 289-336. Washington, D.C.: Dumbarton Oaks Research Library and Collection.

ESTRADA, E., 1956 - Valdivia. Un sitio arqueológico Formativo en la costa de la Provincia del Guayas, Ecuador, 11 p.; Guayaquil: Editorial Vida. Publicación del Museo Víctor Emilio Estrada, 1.

ESTRADA, E., 1961 - Nuevos elementos en la cultura Valdivia: Sus posibles contactos transpacíficos, 14 p.; Guayaquil: Casa de la Cultura Ecuatoriana, Núcleo del Guayas. Publicación del Sub-Comité Ecuatoriano de Antropología, dependiente del Instituto Panamericano de Geografía e Historia.

EVANS, C., MEGGERS, B. J. \& ESTRADA, E., 1959 - Cultura Valdivia, 128 p.; Guayaquil: Editorial Vida. Publicación del Museo Víctor Emilio Estrada, 6.

GRIEDER, T., BUENO M., A., SMITH, C. E. Jr. \& MALINA, R. M., 1988 - La Galgada, Peru. A Preceramic Culture in Transition, 282 p.; Austin: University of Texas Press.

HILL, B. D., 1972-1974 - A New Chronology of the Valdivia Ceramic Complex from the Coastal Zone of Guayas Province. Nawpa Pacha, 10-12: 1-32.

HOOPES, J. W., 1994 - Ford Revisited: A Critical Review of the Chronology and Relationship of the Earliest Ceramic Complexes in the New World, 6000-1500 B.C. Journal of World Prehistory, 8 (1): 1-49.

LEDERGERBER, P., 1983 - El origen de más de un cuarto de siglo de investigaciones sobre la cultura Valdivia. Boletín de la Academia Nacional de Historia, 65: 25-44.

LYON, P. J., 1972-1974 - «Early Formative Period of Coastal Ecuador»: Where is the Evidence? Nawpa Pacha, 10-12: 33-48. 
MARCOS, J. G., 1986 - Breve prehistoria del Ecuador. In: Arqueología de la costa ecuatoriana - Nuevos enfoques (Jorge Marcos, ed.): 25-50; Guayaquil-Quito: ESPOL-Corporación Editora Nacional. Biblioteca Ecuatoriana de Arqueología, 1.

MARCOS, J. G., 1988 - Real Alto. La historia de un centro ceremonial Valdivia (Partes 1-2), 344 p. + 354 p. ; Guayaquil-Quito: ESPOL-Corporación Editora Nacional. Biblioteca Ecuatoriana de Arqueología, 4-5.

MARCOS, J. G., 1998 - A Reassessment of the Chronology of the Ecuadorian Formative. In: El área septentrional andina - arqueología y etnohistoria (Mercedes Guinea, ed.): 295-346; Quito: Abya-Yala. Colección Biblioteca Abya-Yala, 59.

MARCOS, J. G., 2003 - A Reassessment of the Ecuadorian Formative. In: Archaeology of Formative Ecuador. A Symposium at Dumbarton Oaks, 7 y 8 de octubre 1995 (J. Scott Raymond \& Richard L. Burger, eds.): 7-32; Washington, D.C.: Dumbarton Oaks Research Library and Collection.

MARCOS, J. G. \& MICHCZYNSKI, A., 1996 - Good Dates and Bad Dates in Ecuador. Radiocarbon Samples and Archaeological Excavation: A Commentary Based on the «Valdivia Absolute Chronology». In: Problemas de la cronología cultural del área centro-andina. Andes, 1: 93-114; Varsovia: Misión Arqueológica Andina. II Congreso Latinoamericano de la Universidad de Varsovia, Varsovia y Biskupin-Wenecja, 14-17 de junio 1995.

MEGGERS, B. J., 1987 - El origen transpacífico de la cerámica Valdivia: Una revaluación. Boletín del Museo Chileno de Arte Precolombino, 2: 9-31 (2a ed.: Meggers, B. J., 1998 - Evolución y difusión cultural. Enfoques teóricos para la investigación arqueológica, 1. Biblioteca Abya-Yala, 57: 177-226. Quito: Ediciones Abya-Yala).

MEGGERS, B. J., EVANS, C. \& ESTRADA, E., 1965 - Early Formative Period of Coastal Ecuador: The Valdivia and Machalilla Phases, 234 p.; Washington, D.C.: Smithsonian Institution. Smithsonian Contributions to Anthropology, 1.

PORRAS G., P. P. I., 1973 - El Encanto, La Puná: Un sitio insular de la fase Valdivia asociado a un conchero anular, 171 p.; Guayaquil: Museo Francisco Piana. Ediciones Huancavilca 5, Serie La Puná 1.

RAYMOND, J. S., 1999 - Early Formative Societies in the Tropical Lowlands of Western Ecuador: A View from the Valdivia Valley. In: Pacific Latin America in Prehistory. The Evolution of Archaic and Formative Cultures (Michael Blake, ed.): 149-159; Pullman, Wash.: Washington State University Press.

RAYMOND, J. S., 2003 - Social Formations in the Western Lowlands of Ecuador during the Early Formative. In: Archaeology of Formative Ecuador (J. Scott Raymond \& Richard L. Burger, eds.): 33-67; Washington, D.C.: Dumbarton Oaks Research Library and Collection. A Symposium at Dumbarton Oaks, 7 y 8 de octubre 1995.

RAYMOND, J. S., OYUELA-CAYCEDO, A. \& CARMICHAEL, P. H., 1998 - The Earliest Ceramic Technologies of the Northern Andes: A Comparative Analysis. MASCA Research Papers in Science and Archaeology, 15 (Supplement): 153-172.

SCHWARZ, F. A. \& RAYMOND, J. S., 1996 - Formative Settlement Patterns in the Valdivia Valley, SW Coastal Ecuador. Journal of Field Archaeology, 23: 205-224.

SHADY, R., 2003 - El sustento económico del surgimiento de la civilización en el Perú. In: La ciudad sagrada de Caral-Supe. Los orígenes de la civilización andina y la formación del Estado prístino en el antiguo Perú (Ruth Shady \& Carlos Leyva, eds.): 101-105; Lima: Instituto Nacional de Cultura (1a ed. 1999: Boletín del Museo de Arqueología y Antropología de la Universidad Nacional Mayor de San Marcos, 2 (11): 2-4).

STOTHERT, K. E., 1976 - The Early Prehistory of the Sta. Elena Peninsula, Ecuador: Continuities Between Preceramic and Ceramic Cultures. In: Actas del $41^{\circ}$ Congreso Internacional de Americanistas, 2: 88-98; México, D.F.: Instituto Nacional de Antropología e Historia. 41 Congreso Internacional de Americanistas, México, 2 al 7 de septiembre de 1974. 
STOTHERT, K. E., 1988 - La prehistoria temprana de la Península de Santa Elena, Ecuador: Cultura Las Vegas, 271 p.; Guayaquil: Museos del Banco Central del Ecuador. Miscelánea Antropológica Ecuatoriana, Serie Monográfica, 10.

VÉLIZ L., J., s.f. [1971] - Precerámico en Valdivia. s.I. [Guayaquil].

ZEIDLER, J. A., 2003 - Formative Period Chronology for the Coast and Western Lowlands of Ecuador. In: Archaeology of Formative Ecuador (J. Scott Raymond \& Richard L. Burger, eds.): 487-527; Washington, D.C.: Dumbarton Oaks Research Library and Collection. A Symposium at Dumbarton Oaks, 7 y 8 de octubre 1995.

ZIOLKOWSKI, M. S., PAZDUR, M. F., KRZANOWSKI, A. \& MICHCZYNSKI, A., 1994 Andes. Radiocarbon Database for Bolivia, Ecuador and Peru, 604 p.; Warsaw: Andean Archaeological Mission of the Institute of Archaeology, Warsaw University-Gliwice Radiocarbon Laboratory of the Institute of Physics, Silesian Technical University. 